\title{
A CHARACTERIZATION OF FACES OF THE BASE POLYHEDRON ASSOCIATED WITH A SUBMODULAR SYSTEM*
}

\author{
Satoru Fujishige \\ University of Tsukuba
}

(Received May 21, 1983: Final January 23, 1984)

Abstract For a distributive lattice $D \subseteq 2^{E}$ and a submodular function $f$ on $D$ with $\phi \in D$ and $f(\phi)=0$, the pair $(\mathcal{D}, f)$ is called a submodular system and, when $E \in \mathcal{D}$, the polyhedron given by

$$
B(f)=\left\{x \mid x \in R^{E}, \forall X \in D: x(X) \leqq f(X), x(E)=f(E)\right\}
$$

is called the base polyhedron associated with $(D, f)$. We examine the structure of the base polyhedron $B(f)$ and give a characterization of all the faces of $B(f)$. Faces of $B(f)$ are made correspond one-to-one to certain sublattices of $D$, so that the collection $\boldsymbol{D}$ of all such sublattices of $D$ is anti-isomorphic with the collection $\boldsymbol{F}$ of all the nonempty faces of $B(f)$. Here, $\boldsymbol{D}$ and $\boldsymbol{F}$ are considered as posets relative to set inclusion. The incidence relation among faces, dimensions of faces, and extreme points and extreme rays of faces are given based on the structure of the sublattices in $\boldsymbol{D}$. These include as special cases recent results on (1) a poset structure of a polymatroid extreme point and connected components by Bixby, Cunningham and Topkis, (2) extreme rays of a cone determined by a distributive lattice by Tomizawa, and (3) adjacency for polymatroid extreme points by Topkis. Moreover, given a sublattice $D_{1}$ of $D$ on which $f$ is modular,

$$
F\left(D_{1}\right)=\left\{x \mid x \in B(f), \forall X \in D_{1}: x(X)=f(X)\right\}
$$

is a nonempty face of $B(f)$ and there uniquely exists a sublattice $D_{2}$ in $\boldsymbol{D}$ which corresponds to the face $F\left(D_{1}\right)$. We show a theorem which characterizes the relationship between $D_{1}$ and $D_{2} . D_{2}$ is considered as a closure of $D_{1}$ and this closure operation is closely related to the concept of maximal skeleton recently considered by Nakamura and Iri. Algorithmic aspects of these characterizations are also discussed.

\section{Introduction}

Let $E$ be a finite set, $R$ the set of reals, and $D$ a collection of subsets of $E$ which is closed under set union and intersection, i.e., $D$ is

*) The present research was carried out when the author was on leave at Institut für Ökonometrie und Operations Research, Universität Bonn, West Germany, and was supported by the Alexander von Humbold Fellowship $(1982 / 83)$, West Germany. 
a distributive lattice with set union and intersection as the 1attice operations, join and meet. Also let $f: D \rightarrow R$ be a submodular function on $D$, i.e.,

(1.1) $\quad f(X)+f(Y) \geqq f(X \cup Y)+f(X \cap Y)$

for all $X, Y \in D$. (Here, if (1.1) holds with equality for each $X, Y \in D$, $f$ is called a modular function.) Then the pair $(D, f)$ is called a submodular system [6], [8], where we assume $\emptyset \in D$ and $f(\emptyset)=0$. Note that a submodular system is a generalization of the concept of polymatroid [4]. When $E \epsilon$ $D$, the base polyhedron $B(f)$ associated with $(D, f)$ is given by (1.2) $B(f)=\left\{x \mid x \in R^{E}, \forall X \in D: x(X) \leqq f(X), x(E)=f(E)\right\}$.

Here, $R^{E}$ is the set of all real vectors whose coordinates are indexed by $E$ and for a vector $x=(x(e): e \in E) \in R^{E}$ and a set $x \in D$ we define (1.3) $\quad x(x)=\sum_{e \in X} x(e)$

Each vector in $B(f)$ is called a base of $(D, f)$.

In the present paper we shall examine and characterize all the faces of the base polyhedron $B(f)$. In Section 2 we give definitions and preliminary basic results on base polyhedra. Main results of the paper are given in Section 3. Faces of $B(f)$ are made correspond one-to-one to certain sublattices of $D$, so that the collection $\mathbb{D}$ of all such sublattices of $D$ is anti-isomorphic with the collection $\boldsymbol{F}$ of all nonempty faces of $B(f)$. Here, $D$ and $\boldsymbol{F}$ are considered as posets relative to set inclusion. The incidence relation among faces, dimensions of faces, and extreme points and extreme rays of faces are given based on the structure of sublattices in $\mathbb{D}$. These include as special cases recent results on (1) a poset structure of a polymatroid extreme point and connected components by Bixby, Cunningham and Topkis [3], (2) extreme rays of a cone determined by a distributive lattice by Tomizawa [18], and (3) adjacency for polymatroid extreme points by Topkis [21]. Moreover, given a sublattice $D_{1}$ of $D$ on which $f$ is modular, (1.4) $F\left(D_{1}\right)=\left\{x \mid x \in B(f), \forall x \in D_{1}: x(x)=f(x)\right\}$

is a nonempty face of $B(f)$ and there uniquely exists a sublattice $D_{2}$ in $\boldsymbol{D}$ which corresponds to the face $F\left(D_{1}\right)$. We show a theorem which characterizes the relationship between $D_{1}$ and $D_{2} \cdot D_{2}$ is considered as a closure of $D_{1}$ and this closure operation is closely related to the concept of maximal skeleton recent1y considered by Nakamura and Iri [13]. Algorithmic aspects of these characterizations in Section 3 are also discussed in Section 4.

This paper was motivated by the recent work by Bixby, Cunningham and Topkis [3], Nakamura and Iri [13], Tomizawa [18], and Topkis [21]. The importance of submodular functions has now widely been recognized in many combinatorial optimization problems (see, for example, [8], [11], [12], [20]) 
and the present paper will give a further insight into submodular functions.

\section{Definitions and Preliminaries}

In this section we give definitions and preliminaxy results on submodular functions and base polyhedra.

Distributive Lattices and Posets

For a finite set $S$ we denote the cardinality of $S$ by $|S|$ and the set of all the subsets of $S$ by $2^{S}$. Let $D$ be a distributive lattice formed by subsets of a finite set $E$ with set union and intersection as the lattice operations. We assume $\emptyset, E \in D$ throughout the present paper. For such a distributive lattice $D$ there uniquely exists a partially ordered set: (poset) $P(D)=\left(\Pi(D), \leq_{D}\right)$, where $\Pi(D)$ is a partition of $E$ and $\jmath_{0}$ is a partial order on $\Pi(D)$ such that

(2.1) $\quad D=\left\{\tilde{I} \mid I\right.$ is an ideal of the poset $\left.\left(\Pi(D), \preceq_{D}\right)\right\}$ 。

Here, $I \leqq \Pi(D)$ is an ideal of $\left(\Pi(D), \preceq_{D}\right)$ if $T_{1} \preceq_{D} T_{2} \in I$ implies $T_{1} \epsilon$ $I$ for all $T_{1}, T_{2} \in \Pi(D)$, and we define

(2.2) $\quad \tilde{I}=U\{T \mid T \in I\}$

(cf. [2]). (For the construction of the poset $P(D)$, see [6] and [9].) We call $\Pi(D)$ the partition of $E$ induced by $D$. The distributive lattice $D$ is called simple if $\Pi(D)$ is the partition of $E$ into singletons.

For each subset $A$ of $E$ and a partition $P^{\prime}$ of $E$ we say $A$ is compatible with $P^{\prime}$ if for each $T \in P^{\prime}$ either $T \subseteq A$ or $T \subseteq E-A$. For two partitions $P_{1}$ and $P_{2}$ of $E$ we say $P_{1}$ is a refinement of $P_{2}$ if for each $T_{1} \in P_{1}$ there exists $T_{2} \in P_{2}$ such that $T_{1} \leqq T_{2}$.

For two posets $P_{i}=\left(E_{i}, \preceq_{j}\right)(i=1,2)$ we say $P_{2}$ is a homomorphic image of $P_{1}$ if there exists a mapping $\psi$ from $E_{1}$ onto $E_{2}$ such that e $\preceq_{1} e^{\prime}$ implies $\psi(e) \preceq_{2} \psi\left(e^{\prime}\right)$ for all e, é $\epsilon E_{1}$.

operations on Submodular systems

Consider a submodular system $(D, f)$. For a sublattice $D_{0}$ of $D$ we say $f$ is modular on $D_{0}$ if $f$ restricted to $D_{0}$ is a modular function on $D_{0}$. For any $S \in D$ the reduction $(D, f) \cdot S$ of $(D, f)$ to $S$ is the submodular system $\left(D^{S}, f^{S}\right)$ defined by

(2.3) $D^{S}=\{x \mid x \in D, X \subseteq S\}$,

(2.4) $\quad f^{S}(x)=f(x) \quad\left(x \in D^{S}\right)$.

Also the contraction $(D, f) / S$ of $(D, f)$ by $S$ is the submodular system $\left(D_{S}, f_{S}\right)$ defined by

$(2.5) \quad D_{S}=\{X-S \mid S \subseteq X \in D\}$, 


$$
f_{S}(X-S)=f(x)-f(S) \quad(S \subseteq X \in D) .
$$

Any submodular system obtained by repeated reductions and contractions of $(D, f)$ is called a minor of $(D, f)$.

Let $\Pi(D)$ be the partition of $E$ induced by $D$ and $T$ be an element of $\Pi(D)$. Also let $\alpha_{T}$ be a new element not in $E$. Then, define a mapping $\psi: E \rightarrow E^{\prime \prime} \equiv(E-T) \cup\left\{\alpha_{T}\right\}$ by

$$
\text { (2.7) } \psi(e)= \begin{cases}e & (e \in E-T), \\ \alpha_{T} & (e \in T) .\end{cases}
$$

Then $\psi$ naturally induces a distributive lattice $D^{\prime \prime} \subseteq 2^{E^{\prime \prime}}$ and a submodular function $f^{\prime \prime}: D^{\prime \prime} \rightarrow R$, respectively, corresponding to $D$ and $f$. We call $\left(D^{\prime \prime}, f^{\prime \prime}\right)$ the shrinking of $(D, f)$ by $T$. When a partition $P^{\prime}$ of $E$ is a refinement of $\Pi(D)$, we define the shrinking of $(D, f)$ by $P^{\prime}$ as the submodular system obtained by repeated shrinkings of $(\mathcal{D}, f)$ by elements of $P^{\prime}$. If $P^{\prime}=\Pi(D)$, the shrinking of $(D, f)$ by $P^{\prime}$ is called the simplification of $(D, f)$.

For a subset $A$ of $E$ define

$$
D / / A=\{X \mid X \in D \text {, either } A \cap X=\emptyset \text { or } A \subseteq X\}
$$

and let $f / / A$ be the restriction of $f$ to $D / / A$. We call $(D / / A, f / / A$ ) (or $D / / A$ ) the aggregation of $(D, f)$ (or $D)$ by $A$. For a partition $P^{\prime}$ of $E$ the aggregation of $(D, f)$ by $P^{\prime}$ is the submodular system obtained by repeated aggregations of $(D, f)$ by elements of $P^{\prime}$.

We say a submodular system $(D, f)$ is connected if for all nonempty complementary $X, Y \in D$ (i.e., $X \neq \emptyset \neq Y, X=E-Y$ and $X, Y \in D$ ) we have (2.9) $f(X)+f(Y)>f(E)$.

If $(D, f)$ is not connected, there uniquely exists a partition $P^{*}=\left\{T_{1}, T_{2}\right.$, $\left.\ldots, T_{k}\right\}(k \geqq 2)$ of $E$ such that $T_{i} \in D(i=1,2, \ldots, k)$, each reduction $\left(D^{T_{i}}, f^{T_{i}}\right) \equiv(D, f) \cdot T_{i}(i=1,2, \ldots, k) \quad$ is connected, and

$$
f(x)=f^{T}\left(T_{1} \cap X\right)+f^{T} 2\left(T_{2} \cap X\right)+\cdots+f^{T_{k}}\left(T_{k} \cap X\right)
$$

for all $x \in D$. Note that

$$
B=\{X \mid X \in D, E-X \in D, f(X)+f(E-X)=f(E)\}
$$

is a Boolean sublattice of $D$ and $P^{*}=\Pi(B)$. Each $\left(D^{T_{i}}, f^{T_{i}}\right)(i=1,2, \ldots, k)$ is called a connected component of $(D, f)$.

Preliminary Results

Let $(D, f)$ be a submodular system. Based on the general theory of convex polyhedra, we have the following lemma (e.g. [17, p. 67]). For the terminology concerning convex polyhedra we almost follow [17], [14] and [1].

Lemma 2.1. The base polyhedron $B(f)$ can be decomposed as

$$
B(f)=(Q(f)+C(f)) \oplus L(f),
$$


where $Q(f)$ is a bounded polyhedron, $C(f)$ a pointed cone, and $L(f)$ a linear subspace. Here, + denotes a vector sum and $\oplus$ a direct vector sum.

Note that in Lemma $2.1 C(f)$ is unique if and only if $B(f)$ is pointed, i.e., $L(f)=\{0\}$ and that $Q(f)$ is unique if and only if $B(f)$ is bounded, i.e., $L(f)=C(f)=\{0\}$.

Since

$$
L(f)=\left\{x \mid x \in R^{E}, \forall X \in D: x(X)=0\right\},
$$

we can easily show

Lemma 2.2. Suppose that $\left\{T_{i} \mid i \in I\right\}$ is the partition $\Pi(D)$ of $E$ induced by $D$, and define for each $i \in I$

$$
I_{i}(f)=\left\{x \mid x \in R^{E}, x\left(T_{i}\right)=0, \forall e \in E-T_{i}: x(e)=0\right\} .
$$

Then the linear subspace $L(f)$ in Lemma 2.1 is expressed as

$$
L(f)=\underbrace{\oplus}_{i \in I} L_{i}(f) .
$$

Note that $L_{i}(f)=\{0\}$ if and only if $\left|T_{i}\right|=1$. Therefore, $L(f)=$ $\{0\}$, i.e. $B(f)$ is pointed, if and only if $D$ is simple. The simplification of $(D, f)$ determines the structure of $Q(f)$ and $C(f)$ in Lemma 2.1, since one possible $Q(f)+C(f)$ is given by

$$
Q(f)+C(f)=\left\{x \mid x \in B(f), \forall e \in E-E^{*}: x(e)=0\right\},
$$

where $E^{*}=\left\{e_{i} \mid i \in I\right\}$ with $e_{i} \in T_{i}$ (i $\left.\in I\right)$, i.e., $E^{*}$ is a set of representatives of $T_{i}(i \in I)$ in $\Pi(D)$.

When $D$ is simple, $C(f)$ is unique and one possible $Q(f)$ is given by the convex hull of all the extreme points of $B(f)$. The following theorem characterizes the extreme points of $B(f)$.

Theorem 2.3 [9] (when $D=2^{E}$ see [4], [12], [16]). When $D$ is simple, a base $b \in B(f)$ is an extreme point of $B(f)$ if and only if for a maximal chain

$$
C: \emptyset=S_{0} \varsubsetneqq S_{1 \nsupseteq} \cdots \varsubsetneqq S_{n}=E
$$

in $D$ we have

$$
b\left(S_{i}-S_{i-1}\right)=f\left(S_{i}\right)-f\left(S_{i-1}\right) \quad(i=1,2, \ldots, n) .
$$

Denote by $H(P(D))=\left(\Pi(D), A^{*}(D)\right)$ the Hasse diagram representing the poset $P(D)=(\Pi(D), \preceq D)$. Here, $H(P(D))$ is a directed graph with a vertex set $\Pi(D)$ and an arc set $A^{*}(D)$ such that $a \in A^{*}(D)$ if and only if $\partial^{-} a$ $\prec_{0} \partial^{+} a$ and there is no element $T \in \Pi(D)$ with $\partial^{-} a \prec_{D} \tau_{0} \partial^{+} a$, where $\partial^{2} a\left(\partial^{+} a\right) \in \Pi(D)$ is the terminal (initial) end-vertex of arc $a$. When $D$ is simple, we express $P(D)=\left(\Pi(D), \preceq_{D}\right)$ and $H(P(D))=\left(\Pi(D), A^{*}(D)\right)$, respectively, as $P(D)=\left(E, \preceq_{D}\right)$ and $H(P(D))=\left(E, A^{*}(D)\right)$ as well, since $\Pi(D)$ 
is the partition of $E$ into singletons.

Now, from the general theory [14], [17], the recession cone $C(f)$ of $B(f)$ is expressed as

$$
C(f)=\left\{x \mid x \in R^{E}, \forall x \in D: x(x) \leqq 0, x(E)=0\right\} .
$$

(The term, characteristic cone, is used in [17] instead of recession cone.) The following theorem determines the extreme rays of $C(f)$.

Theorem 2.4 [18]. Suppose $D$ is simple. Then the set of all the extreme rays of $C(f)$ is given by

$$
\{\zeta(a) \mid a \in A *(D)\} \text {, }
$$

where $A^{*}(D)$ is the arc set of the Hasse diagram $H(P(D))=\left(E, A^{*}(D)\right)$ and $\zeta(a)$ is the vector in $R^{E}$ defined by

$$
\zeta(a)(e)=\left\{\begin{array}{rl}
1 & \left(e=\partial^{+} a\right) \\
-1 & \left(e=\partial^{-} a\right) \\
0 & \text { (otherwise) }
\end{array} \quad(e \in E) .\right.
$$

In the next section we shall give a characterization of all the faces of $B(f)$ when $D$ is simple.

\section{A Characterization of Faces of the Base Polyhedron}

We assume that $D \subseteq 2^{E}$ is a simple distributive lattice with $\emptyset, E \in D$ and that $f: D \rightarrow R$ is a submodular function with $f(\emptyset)=0$.

For any $F \subseteq D$ define

(3.1) $F(F)=\left\{x \mid x \in R^{E}, \forall x \in F: x(x)=f(x), \forall x \in D-F: x(x) \leq f(x)\right\}$, (3.2) $F^{\circ}(F)=\left\{x \mid x \in R^{E}, \forall x \in F: x(x)=f(x), \forall x \in D-F: x(x)<f(x)\right\}$. Moreover, define

$$
\mathbb{D}=\left\{D_{0} \mid D_{0} \text { is a sublattice of } D \text { with } \emptyset, E \in D_{0}, F^{\circ}\left(D_{0}\right) \neq \emptyset\right\} .
$$

Lemma 3.1. The collection $\mathbb{D}$ of sublattices of $D$ defined by (3.3) is given by

$$
D=\{D(x) \mid x \in B(f)\},
$$

where for each $x \in B(f)$

$$
D(x)=\{x \mid x \in D, x(X)=f(X)\} \text {. }
$$

Proof: If $D_{0} \in \mathbb{D}$, then for some $x \in F^{\circ}\left(D_{0}\right)$ we must have $D_{0}=D(x)$ from (3.2). Conversely, it is easily seen that for any $x \in B(f) D(x)$ is a sublattice of $D$ with $\emptyset, E \in D(x)$ and $x \in F^{\circ}(D(x))$, and we have $D(x) \epsilon$ D. Q.E.D.

It may be noted that the poset induced by $D(x)$ in (3.5) is what is called the principal structure of $(D, f-x)$ in [6], where considered is the 
case when $D=2^{E}$. It should also be noted that for $D_{0} \in D F^{\circ}\left(D_{0}\right)$ is the relative interior of the face $F\left(\mathcal{D}_{0}\right)$.

From Lemma $3.1 \boldsymbol{D}$ is the collection of "equality sets", each given by (3.5) (cf. [1]), for $B(f)$ expressed by (1.2). Therefore, from the general theory of convex polyhedra [1], [17] we have the following

Theorem 3.2. The collection $\boldsymbol{F}$ of al1 the nonempty faces of $B(f)$ is given by $\left.\left\{F\left(D_{0}\right) \mid D_{0} \in \mathbb{I}\right)\right\}$, and we have

(i) If $\left.D_{1}, D_{2} \in \mathbb{I}\right)$ and $D_{1} \neq D_{2}$, then $F\left(D_{1}\right) \neq F\left(D_{2}\right)$.

(ii) For any $D_{1}, D_{2} \in \mathbb{D}, D_{1} \subseteq D_{2}$ if and only if $F\left(D_{2}\right) \cong F\left(D_{1}\right)$. In other words, $F$ in (3.1) determines an anti-isomorphism from $\boldsymbol{D}$ onto $\boldsymbol{F}$, where $\mathbb{D}$ and $\boldsymbol{F}$ are considered as posets relative to set inclusion.

$\boldsymbol{F}($ or $\boldsymbol{F} \cup\{\emptyset\}$ when $\boldsymbol{F}$ does not have a unique minimal element) is called the face lattice of $B(f)$ (see [1], [17]).

Lemma 3.3. For any distributive lattices $D_{i}(i=1,2)$ with $\emptyset, E \in D_{i}$, we have $D_{1} \subseteq D_{2}$ if and only if $\Pi\left(D_{2}\right)$ is a refinement of $\Pi\left(D_{1}\right)$ and $P\left(D_{1}\right)=\left(\Pi\left(D_{1}\right), \preceq_{D_{1}}\right)$ is a homomorphic image of $P\left(D_{2}\right)=\left(\Pi\left(D_{2}\right), \preceq_{D_{2}}\right)$ under the natural mapping (i.e., $T_{2} \in \Pi\left(D_{2}\right)$ is made correspond to $T_{1} \in \Pi\left(D_{1}\right)$ if $\left.T_{2} \subseteq T_{1}\right)$.

Proof: The lemma follows from the following:

(1) $\Pi\left(D_{1}\right)$ is the collection of equivalence classes with respect to the equivalence relation $\tau_{1}$ such that $e \tau_{1} e^{\prime}$ if and only if there exists no $X \in D_{1}$ with $e \in X \neq e^{\prime}$ or $e \notin X \ni e^{\prime}$, and

(2) $T \bigcirc_{1} T^{\prime}$ if and only if $T^{\prime} \leqq X \in D_{1}$ implies $T \subseteq X$ for all $X \epsilon$ $D_{1}$, where $T, T^{\prime} \in \Pi\left(D_{1}\right)$.

Q.E.D.

Theorem 3.4. For $D_{0} \in \mathbb{D}$ we have

$$
\operatorname{dim} F\left(D_{0}\right)=|E|-\left|\Pi\left(D_{0}\right)\right| \text {, }
$$

where $\operatorname{dim} F\left(D_{0}\right)$ denotes the dimension of the face $F\left(D_{0}\right)$.

Proof: The dimension of the face $F\left(D_{0}\right)$ is equal to that of the affine set

$$
M\left(D_{0}\right)=\left\{x \mid x \in R^{E}, \forall x \in D_{0}: x(x)=f(x)\right\} .
$$

Since the rank of the coefficient matrix in the right-hand side of (3.7) is equal to $\left|\Pi\left(D_{0}\right)\right|$, we have $(3.6)$.

Q.E.D.

It may be noted that Theorems 2.3 and 2.4 easily follow from Theorems 3.2 and 3.4 and Lemma 3.3 .

Lemma 3.5. Suppose $D_{0} \in \mathbb{D}$ and let

$$
C_{0}: \emptyset=s_{0 \varsubsetneqq} s_{1 \varsubsetneqq} \cdots c_{k} s_{k}=E
$$

be a maximal chain in $\mathcal{D}_{0}$. Then, 
(3.9)

$$
F\left(C_{0}\right)=F\left(D_{0}\right)
$$

Proof: It suffices to prove that $F\left(C_{0}\right) \subseteq F\left(D_{0}\right)$. For any $a \in F\left(C_{0}\right)$ and $b \in F\left(D_{0}\right)$ we have

$$
\begin{aligned}
& a\left(S_{i}-S_{i-1}\right)=b\left(S_{i}-S_{i-1}\right) \quad(i=1,2, \ldots, k), \\
& \left\{S_{i}-S_{i-1} \mid i=1,2, \ldots, k\right\}=\Pi\left(D_{0}\right),
\end{aligned}
$$

since $C_{0}$ is a maximal chain in $D_{0}$. Therefore, for any $x \in D_{0}$

(3.12) $a(x)=b(x)=f(x)$, i.e.,

$$
a \in F\left(D_{0}\right)
$$

The following theorem characterizes the extreme points of a face of $B(f)$.

Theorem 3.6. Suppose $D_{0} \in \mathbb{D}$. Then a base $b \in B(f)$ is an extreme point of the face $F\left(D_{0}\right)$ if and only if, for a maximal chain

$$
\mathcal{C}: \emptyset=s_{0} \subsetneq S_{1} \subsetneq \cdots \subsetneq S_{n}=E
$$

in $D$ which contains a maximal chain in $D_{0}$ as a subchain, $b$ is given by (3.14) $b\left(s_{i}-S_{i-1}\right)=f\left(s_{i}\right)-f\left(S_{i-1}\right) \quad(i=1,2, \ldots, n)$.

Proof: "If" part. Let $C_{0}$ be a maximal chain in $D_{0}$ contained in (3.13). Then from (3.13) and (3.14) we have $b \in F\left(C_{0}\right)$. It follows from Lemma 3.5 that $b \in F\left(D_{0}\right)$. Moreover, from Theorem $2.3 b$ is an extreme point of $B(f)$. Consequently, $b$ must be an extreme point of $F\left(D_{0}\right)$.

"Only if" part. For any extreme point $b$ of $F\left(D_{0}\right)$, define $D(b)$ by (3.5). Then, $D_{0} \subseteq D(b) \in \mathbb{D}$. Since $b$ is also an extreme point of $B(f)$, i.e., $\{b\}$ is a zero-dimensional face $F(D(b))$ of $B(f)$, a maximal chain in $D(b)$ is a maximal chain in $D$ due to Theorem 3.4 (or Theorem 2.3) and there exists a maximal chain in $D(b)$ which contains a maximal chain in $D_{0}$ as a subchain.

Extreme rays of a face of $B(f)$ are characterized by the following

Theorem 3.7. Suppose $D_{0} \in \mathbb{D}$ and $\Pi\left(D_{0}\right)=\left\{T_{i} \mid i \in I\right\}$. Then the set of all the extreme rays of the recession cone of the face $F\left(D_{0}\right)$ is given by (3.15) $\left\{\zeta(a) \mid a \in A^{*}(D), \exists i \in I:\left\{\partial^{+} a, \partial^{-} a\right\} \subseteq T_{i}\right\}$, where $A^{*}(D)$ is the arc set of the Hasse diagram $H(P(D))=\left(E, A^{*}(D)\right)$ representing the poset $P(D)=\left(E, \preceq_{D}\right)$ and $\zeta(a)$ is defined by $(2.21)$.

Proof: The present theorem easily follows from Theorem 2.4 and the fact that the recession cone $C\left(D_{0}\right)$ of the face $F\left(D_{0}\right)$ is given by

$$
C\left(D_{0}\right)=\left\{x \mid x \in R^{E}, \forall x \in D_{0}: x(x)=0, \forall x \in D-D_{0}: x(x) \leqq 0\right\} .
$$


Now, let us consider the problem of finding an element $D_{0} \in \mathbb{D}$ for which $B(f)=F\left(D_{0}\right)$.

Theorem 3.8. A submodular system $(\mathcal{D}, f)$ is connected if and only if $\{\emptyset, E\} \in \mathbb{D}$, i.e., there exists a base $b \in B(f)$ such that

$$
b(x)<f(X)
$$

for any $X \in D$ with $\emptyset \neq X \neq E$.

Proof: "If" part. Easy. (Note that $b(E)=f(E)$ for $b \in B(f)$.)

"OnIy if" part. Suppose $(D, f)$ is connected and $B(f)=F\left(D_{0}\right)$ for some $D_{0} \in \mathbb{D}$. We show $D_{0}=\{\emptyset, E\}$. Suppose, on the contrary, that there were $Y_{0} \in D_{0}$ with $\emptyset \neq Y_{0} \neq E$. Then the inequality

(3.18) $-x\left(y_{0}\right) \leqq-f\left(y_{0}\right)$

must be implied by the following system of inequalities and an equation:

(3.19) $\quad x(X) \leqq f(X) \quad(X \in D-\{E\})$,

(3.20) $x(E)=f(E)$.

Therefore, there exist rational $\alpha(X) \geqq 0(X \in D-\{E\})$ and rational $\alpha(E)$ $<0$ such that

$$
\begin{aligned}
(3.21)-X\left(Y_{0}\right) & =\sum_{X \in D} \alpha(X) x(x), \\
(3.22)-f\left(Y_{0}\right) & \geqq \sum_{X \in D} \alpha(x) f(x),
\end{aligned}
$$

where $X(X)$ for each $X \in D$ is the characteristic vector of $X \subseteq E$. Define (3.23) $F=\{X \mid X \in \mathcal{D}, \alpha(X)>0\}$.

If $F$ contains incomparable $x_{1}, x_{2}$ (relative to set inclusion), then for $\varepsilon \equiv \min \left(\alpha\left(x_{1}\right), \alpha\left(x_{2}\right)\right)$ put
(3.24) $\alpha\left(x_{1}\right) \leftarrow \alpha\left(x_{1}\right)-\varepsilon$,
(3.25) $\alpha\left(x_{2}\right) \leftarrow \alpha\left(x_{2}\right)-\varepsilon$,
(3.26) $\quad \alpha\left(x_{1} \cup x_{2}\right) \leftarrow \alpha\left(x_{1} \cup x_{2}\right)+\varepsilon$,
(3.27) $\alpha\left(x_{1} \cap x_{2}\right) \leftarrow \alpha\left(x_{1} \cap x_{2}\right)+\varepsilon$.

Repeat the above (3.24) $\sim(3.27)$ until $F$ given by (3.23) consists of pairwise comparable elements of $D$. After a finite number of steps this process terminates. Note that throughout this process (3.21) and (3.22) hold because of $(3.24) \sim(3.27)$ and the submodularity of $f$. For the finally obtained $F$ and $\alpha$ we must have

(3.28) $F=\left\{E-Y_{0}\right\}$ or $\left\{\emptyset, E-Y_{0}\right\}$,

(3.29) $\alpha\left(E-Y_{0}\right)=1, \quad \alpha(E)=-1$

because of (3.21) with $D$ replaced by $F \cup\{E\}$. Therefore,

(3.30) $E-Y_{0} \in D$.

Also we have from $(3.22) \sim(3.29)$

(3.31) $-f\left(Y_{0}\right) \geqq f\left(E-Y_{0}\right)-f(E)$, 
or

(3.32) $f\left(Y_{0}\right)+f\left(E-Y_{0}\right) \leqq f(E)$.

Consequent1y,

(3.33) $\quad f\left(Y_{0}\right)+f\left(E-Y_{0}\right)=f(E)$,

which contradicts the connectedness of $(D, f)$.

Q.E.D.

Theorem 3.9. Suppose $D_{0} \in \mathbb{D}$ and $B(f)=F\left(D_{0}\right)$. Then $D_{0}$ is a Boolean sublattice of $D$ and $\Pi\left(D_{0}\right)$ is the partition of $E$ given by the decomposition of $(D, f)$ into connected components. In particular, the number of the connected components is equal to $\left|\Pi\left(D_{0}\right)\right|$.

Proof: It follows from the proof of Theorem 3.8 that if $B(f)=F\left(D_{0}\right)$ for any $D_{0} \in \mathbb{D}$ and $Y_{0} \in D_{0}$, then we have $E-Y_{0} \in D_{0}$. Theorefore, $D_{0}$ is a complemented distributive lattice, i.e., a Boolean lattice. The rest of the theorem follows from the definition of a connected component. Q.E.D.

From Theorems 3.4 and 3.9 we have

Corollary 3.10. Let $n^{*}$ be the number of connected components of $(D, f)$. Then,

(3.34) $\operatorname{dim} B(f)=|E|-n^{*}$.

The dimension of $B(f)$ was given in [16], [10], [3] (when $D=2^{E}$ ) and [19]. The connected components of $(D, f)$ is efficiently found by an algorithm in [3].

Corollary 3.11. Let $D_{1}$ be a sublattice of $D$ with $\emptyset, E \in D_{1}$ and suppose $f$ is modular on $D_{1}$. Also let

(3.35) $\quad c_{1}: \emptyset=s_{0 \nsubseteq} S_{1 \nsucceq} \cdots S_{k}=E$

be any maximal chain in $D_{1}$ and for each $i=1,2, \ldots, k$ let $n_{i} *$ be the number of connected components of the minor $(D, f) \cdot S_{i} / s_{i-1}$. Then the dimension of the face $F\left(D_{1}\right)$ is given by

$$
\operatorname{dim} F\left(D_{1}\right)=|E|-\sum_{i=1}^{\mathrm{k}} n_{i}^{*} .
$$

Proof: Note that since $f$ is modular on $D_{1}, x \in F\left(D_{1}\right)$ if and only if for each $i=1,2, \ldots, k \quad x$ restricted to $S_{i}-S_{i-1}$ is a base of $(D, f) \cdot S_{i} / S_{i-1}$. Therefore, the present corollary follows from Corollary 3.10.

Corollary 3.11 when $D=2^{E}$ and $D_{1}$ is a chain of length 2 is also shown in [10]. Note that $F\left(D_{1}\right) \neq \emptyset$ if and only if $f$ is modular on $D_{1}$. Also note that in Corollary 3.11 since $f$ is modular on $D_{1}$, for each $T \in$ $\Pi\left(D_{1}\right)$ the minor $(D, f) \cdot S_{i} / S_{i-1}$ with $S_{i}-S_{i-1}=T$ does not depend on the 
choice of a maximal chain (3.35) in $D_{1}$ (cf. [13], [20]).

Theorem 3.12. For any $D_{1}, D_{2} \in \mathbb{D}$ we have $D_{1} \cap D_{2} \in \mathbb{D}$ and $F\left(D_{1} \cap D_{2}\right)$ is the minimal face (relative to set inclusion) which contains both $F\left(D_{1}\right)$ and $F\left(D_{2}\right)$.

Proof: Suppose $D_{\prod}, D_{2} \in \mathbb{D}$. Since $D_{1} \cap D_{2}$ is a sublattice of $D$ with $\emptyset, E \in D_{1} \cap D_{2}$, it suffices to show $F^{\circ}\left(D_{1} \cap D_{2}\right) \neq \emptyset$. By the assumption there exist $b_{i} \in F^{\circ}\left(D_{i}\right)(i=1,2)$. For an arbitrary $\lambda \in R$ with $0<\lambda<1$, $\lambda b_{1}+(1-\lambda) b_{2} \in F^{\circ}\left(D_{1} \cap D_{2}\right)$.

The minimality of the face follows from Theorem 3.2.

Q.E.D.

Theorem 3.12 also follows from the general theory for convex polyhedra (cf. [1]).

If $D_{1}, D_{2} \in \mathbb{D}$ and $F\left(D_{1} \cup D_{2}\right) \neq \emptyset$, then $F\left(D_{1} \cup D_{2}\right)$ is the unique maximal face of $B(f)$ which is contained in both $F\left(D_{1}\right)$ and $F\left(D_{2}\right)$ and there exists $D_{3} \in D$ such that $F\left(D_{1} \cup D_{2}\right)=F\left(D_{3}\right)$. Let $D_{1} \vee D_{2}$ be the unique minimal sublattice of $D$ (minimal relative to set inclusion) which contains both $D_{1}$ and $D_{2}$. Then $D_{1} \vee D_{2} \cong D_{3}$. Note that $D_{3}$ is the unique minimal sublattice of $D$ in $\mathbb{D}$ which contains both $D_{1}$ and $D_{2}$.

The following theorem gives a necessary and sufficient condition for a sublattice $D_{0}$ of $D$ to be a member of $\mathbb{D}$.

Theorem 3.13. Let $D_{0}$ be any sublattice of $D$ with $\emptyset, E \in D_{0}$ and $f_{0}$ be the restriction of $f$ to $D_{0}$. Then $D_{0} \in \mathbb{D}$ if and only if the following three statements hold:

(i) $f_{0}$ is a modular function.

(ii) For any maximal chain

(3.38) $\quad C_{0}: \emptyset=S_{0} \subsetneq S_{1} \subsetneq \cdots \subsetneq S_{k}=E$

in $D_{0}$ each minor $(D, f) \cdot S_{i} / S_{i-1}(i=1,2, \ldots, k)$ is connected.

(iii) Let $C_{0}$ be any maximal chain in $D_{0}$ as in (3.38) and $\hat{b}$ be any base of $\left(D_{0}, f_{0}\right)$ such that

$$
\hat{b}\left(s_{i}\right)=f_{0}\left(S_{i}\right) \quad(i=1,2, \ldots, k) .
$$

Then for any $x \in D$ such that $x$ is compatible with $\Pi\left(D_{0}\right)$ and $\hat{b}(x)=$ $f(x)$, we have $x \in D_{0}$.

Proof: "If" part. Suppose (i) (iii) hold. For each $i=1,2, \ldots$, $k$ let $b_{i}^{*}$ be a base of $\left(D_{i}, F_{i}\right) \equiv(D, f) \cdot S_{i} / S_{i-1}$ such that for any $x \in D$ with $s_{i-1} \subsetneq{ }_{\ddagger} S_{i}$

(3.40) $\quad b_{i}^{*}\left(x-S_{i-1}\right)<f(x)-f\left(S_{i-1}\right)=f_{i}\left(x-S_{i-1}\right)$.

Such a base $b_{i}^{*}$ exists due to Theorem 3.8. Define $b^{*} \in R^{E}$ by (3.41) $\quad b^{*}(e)=b_{i} *(e)$

for each $e \in E$ with $e \in S_{i}-S_{i-1}$ and $i \in\{1,2, \ldots, k\}$. Then $b^{*} \in B(f)$. 
We show $b^{*} \in F^{\circ}\left(D_{0}\right)$, which will complete the proof of the "if" part. Suppose that there were $x_{0} \in \mathcal{D}$ such that (3.42) $\quad b^{*}\left(x_{0}\right)=f\left(x_{0}\right)$

and for some $T \in \Pi\left(D_{0}\right)$

$$
X_{0} \cap T \neq \emptyset, \quad T-X_{0} \neq \emptyset \text {. }
$$

Suppose $T=S_{i_{0}}-S_{i_{0}-1}$. By the definition of $b^{*}$,

(3.44) $\quad b^{*}\left(S_{i}\right)=f\left(S_{i}\right) \quad(i=1,2, \ldots, k)$.

It follows from (3.42) and (3.44) with $i=i_{0}-1, i_{0}$ that for $Y_{0} \equiv\left(x_{0} u\right.$ $\left.S_{i_{0}-1}\right) \cap S_{i_{0}}$ we have

(3.45) $\quad b^{*}\left(Y_{0}\right)=f\left(Y_{0}\right)$,

since $D\left(b^{*}\right)=\left\{x \mid x \in D, b^{*}(X)=f(X)\right\}$ is a sublattice of $D$. However, because of Theorem 3.8 (3.45) contradicts that $\left(D_{i_{0}}, f_{i_{0}}\right)$ is connected, since $S_{i_{0}} \varsubsetneqq Y_{0} \subsetneq S_{i_{0}}$ from (3.43). Therefore, if $b^{*}\left(X_{0}\right)=f\left(x_{0}\right)$ for any $x_{0} \in D, x_{0}$ does not satisfy (3.43) for any $T \in \Pi\left(D_{0}\right)$, i.e., $x_{0}$ is compatible with $\Pi\left(D_{0}\right)$. Then, from (iii) we have $x_{0} \in D_{0}$. Consequently, (3.46) $\quad b^{*}(x)<f(x) \quad\left(x \in D-D_{0}\right)$.

Furthermore, because of $(i)$ and (3.44)

(3.47) $\quad b^{*}(X)=f(X) \quad\left(X \in D_{0}\right)$.

It follows from (3.46) and (3.47) that $b^{*} \in F^{\circ}\left(D_{0}\right)$.

"Only if" part. Suppose $D_{0} \in \mathbb{D}$. Then there exists a vector $b^{\prime} \epsilon$ $F^{\circ}\left(D_{0}\right)$ and $(i)$ is immediate. Also (ii) follows from Theorem 3.8. We show (iii). Let $\hat{b}$ be any base of $\left(D_{0}, f_{0}\right)$ such that (3.39) holds. Suppose that $x_{0} \in D$ is compatible with $\Pi\left(D_{0}\right)$ and

(3.48) $\hat{b}\left(x_{0}\right)=f\left(x_{0}\right)$.

Since $b^{\prime} \in F^{\circ}\left(D_{0}\right)$ also satisfies

(3.49) $\quad b^{\prime}\left(s_{i}\right)=f_{0}\left(s_{i}\right) . \quad(i=1,2, \ldots, k)$,

we have from (3.39) and (3.49)

(3.50)

$\hat{b}(T)=b^{\prime}(T)$

$\left(T \in \Pi\left(D_{0}\right)\right)$.

Since $x_{0}$ is compatible with $\Pi\left(D_{0}\right)$, we have from (3.48) and (3.50)

(3.51) $\quad b^{\prime}\left(x_{0}\right)=\hat{b}\left(x_{0}\right)=f\left(x_{0}\right)$.

Therefore, since $b^{\prime} \in F^{\circ}\left(D_{0}\right)$, we must have $x_{0} \in D_{0}$. Q.E.D.

From Theorems 3.4 and 3.13 we have the following

Corollary 3.14. Suppose that $B(f)$ is full-dimensional, i.e., $\operatorname{dim} B(f)$ $=|E|-1$ and let $D_{0}$ be a sublattice of $D$ with $\emptyset, E \in D_{0}$. Then, $F\left(D_{0}\right)$ is a facet of $B(f)$ if and only if $D_{0}$ forms a chain $\emptyset=s_{0} \varsubsetneqq S_{1} \varsubsetneqq S_{2}=\mathrm{E}$ in $D$ of length 2 and $(D, f) \cdot S_{i} / S_{i-1}(i=1,2)$ are connected.

We also have 
Corollary 3.15. Let $D_{1}$ be a sublattice of $D$ with $\emptyset, E \in D_{1}$ and suppose $f$ is modular on $D_{1}$. Also let (3.52) $\quad C_{1}: \emptyset=S_{0} \subsetneq S_{1} \subsetneq \cdots \varsubsetneqq S_{k}=E$ be any maximal chain in $D_{1}$ and for each $i=1,2, \ldots, k$ let $P_{i}=\left\{T_{i}^{1}\right.$, $\left.T_{i}{ }^{2}, \ldots, T_{i}{ }^{S_{i}}\right\}$ be a partition of $S_{i}-S_{i-1}$ given by the decomposition of $(D, f) \cdot S_{i} / S_{i-1}$ into connected components. Define $P^{*}=U_{i=1}^{k} P_{i}$ and let $\hat{b}$ be a base of $(D, f)$ such that

$$
\hat{b}\left(S_{i}\right)=f\left(S_{i}\right) \quad(i=1,2, \ldots, k) .
$$

Then $D_{2}$ given by

(3.54) $\quad D_{2}=\left\{x \mid x \in D, \hat{b}(x)=f(x), x\right.$ is compatible with $\left.P^{*}\right\}$

belongs to $\mathbb{D}$ and satisfies

(3.55) $\quad F\left(D_{1}\right)=F\left(D_{2}\right)$.

Proof: It is easy to see that $D_{2}$ given by (3.54) satisfies (i) (iii) of Theorem 3.13, and $D_{2} \in \mathbb{D}$. Moreover, for any $b^{\prime} \in F\left(D_{1}\right)$ we have (3.56) $\hat{b}\left(T_{i}^{j}\right)=b^{\prime}\left(T_{i}{ }^{j}\right)$

for each $j=1,2, \ldots, s_{i}$ and $i=1,2, \ldots, k$. Consequently, (3.57) $\quad b^{\prime}(x)=\hat{b}(x)=f(x)$

for any $x \in D_{2}$.

Q.E.D.

Note that the operation of getting $D_{2}$ from $D_{1}$ in Corollary 3.15 is a closure operation on sublattices of $D$ containing $\emptyset$ and $E$, and $D$ is the collection of closed sublattices of $D$ containing $\emptyset$ and $E$.

It should also be noted that Corollary 3.15 is closely related to the concept of $f$-skeleton introduced in [13]. An f-skeleton is a sublattice of $D$ on which $f$ is modular. A sublattice $D_{1}$ of $D$ in Corollary 3.15 is an f-skeleton and the exists a unique maximal $f$-skeleton $D_{1}$ * (maximal relative to set inclusion) such that $\Pi\left(D_{1}^{*}\right)=\Pi\left(D_{1}\right)$, which is called the maximal f-skeleton corresponding to $D_{1}$ (see [13]). We can easily see that $D_{1}$ * may not be closed and $D_{1}{ }^{\circ} \subseteq D_{2}$, where $D_{2}$ is the closure of $D_{1}$ given by (3.54). In fact, $D_{1}{ }^{*}$ is the aggregation of $D_{2}$ by $\Pi\left(D_{1}\right)$. Therefore, $D_{2}$ is a finer structure than the maximal f-skeleton $D_{1}{ }^{*}$. Corollary 3.15 gives a polyhedral interpretation of skeletons.

\section{Remarks on Algorithmic Aspects and Related Topics}

Based on Theorem 3.13 we can solve the following problem in polynomial time (with respect to $|E|$ using an oracle for function evaluation of $f$ ): Given a sublattice $D_{0}$ of $D$, decide whether $D_{0} \in \mathbb{D}$, where we assume that: the corresponding posets $P\left(D_{0}\right)$ and $P(D)$ are given in the form of the Hasse 
diagrams. (ii) in Theorem 3.13 is recognized by using an algorithm proposed in [3]. (i) and (iii) are recognized as follows. Consider the simplification $\left(D_{0}{ }^{\prime}, f_{0}^{\prime}\right)$ of $\left(D_{0}, f_{0}\right)$ and find an extreme base $b_{0}$ of $\left(D_{0}^{\prime}, f_{0}^{\prime}\right)$. Then (i) holds if and only if for all e $\in E^{\prime} \equiv \Pi\left(D_{0}\right)$ $b_{0}(e)=f_{0}^{\prime}(D(e))-f_{0}^{\prime}(D(e)-\{e\})$,

where $D(e)$ is the unique minimal element of $D_{0}^{\prime}$ which contains $e$. It is easy to check (4.1). (A similar method for recognizing a modular function when $D=2^{E}$ was also communicated by Tomizawa.) Let $\left(D_{1}, f_{1}\right)$ be the aggregation of $(D, f)$ by $\Pi\left(D_{0}\right)$ and further let $\left(D_{1}^{\prime}, f_{1}^{\prime}\right)$ be the simplification of $\left(D_{1}, f_{1}\right)$, where $D_{0}^{\prime} \subseteq D_{1}^{\prime}$. Then (iii) holds if and only if (4.2) $D_{0}^{\prime}=\left\{x \mid x \in D_{1}^{\prime}, b_{0}(x)=f_{1}^{\prime}(x)\right\}$.

Relation (4.2) is recognized by the use of the algorithm in [3] for determining the poset associated with $D_{0}$.

In a similar way we can find in polynomial time in $|E|$ the sublattice $D_{2}$, the closure of $D_{1}$, in Corollary 3.15 for a given sublattice $D_{1}$ of $D$. Given a fixed integer $p \geqq 1$, we can decide, in polynomial time in $|E|$, whether a given vector $b \in R$ belongs to a face of $B(f)$ whose dimension is less than $p$. This follows from the fact that if $b \in B(f)$, then $D(b)$ geven by (3.5) belongs to $\mathbb{D}, F(D(b))$ is the minimal face of $B(f)$ which contains $b$, and if $\operatorname{dim} F(D(b))<p$, we have $|T| \leqq p$ for each $T \in \Pi(D(b))$. A polynomial algorithm is given by a direct adaptation of the algorithm in [3] for determining whether a vector in $R^{E}$ is an extreme point of $B(f)$.

Based on Theorems 3.4 and 3.13 and Lemma 3.3, the incidence relation between extreme points and edges (one-dimensional faces) of $B(f)$ is given as follows:

"Let $b$ be an extreme point of $B(f)$ and $K$ be an edge of $B(f)$. Suppose $\{b\}=F\left(D_{0}\right)$ and $K=F\left(D_{1}\right)$ for some $D_{0}, D_{1} \in \mathbb{D}$. Then, $\left|\Pi\left(D_{0}\right)\right|=|E|$ and $\left|\Pi\left(D_{1}\right)\right|=|E|-1$. Moreover, $b \in K$ if and only if $D_{1} \subseteq D_{0}$ and for $T \in \Pi\left(D_{1}\right)$ with $|T|=2$ and $S_{1}, S_{2} \in \Pi\left(D_{1}\right)$ with $S_{1} \subsetneq S_{2}$ and $T=S_{2}-S_{1} \quad(D, f) \cdot S_{2} / S_{1}$ is connected."

In other words, $b \in K$ if and only if for $\left\{e, e^{\prime}\right\} \in \Pi\left(D_{1}\right)$ vertices $e$ and $e^{\prime}$ in the Hasse diagram $H\left(P\left(D_{0}\right)\right)=\left(E, A^{*}\left(D_{0}\right)\right)$ is connected by an arc in $A^{*}\left(D_{0}\right)$ and $H\left(P\left(D_{1}\right)\right)$ is obtained by identifying $e$ with $e^{\prime}$ in $H\left(P\left(D_{0}\right)\right)$. The incidence relation between extreme points and edges of $B(f)$ can also be derived from Theorem 2.4 (cf. [19]). Consequently, the number of edges incident with a common extreme point is equal to the number of arcs of the Hasse diagram associated with the extreme point, which is bounded by
$|E|^{2} / 4$
(if $|E|$ is even),
$\left(|E|^{2}-1\right) / 4$
(if
$|E|$ is odd). 
Recent1y, Topkis [21] has given a characterization of adjacency for extreme points of a polymatroid polytope (a polytope of independent vectors of a polymatroid). For a submodular system $(D, f)$ adjacency for extreme points of the base polyhedron $B(f)$ is characterized as follows, based on Theorems 3.4 and 3.13 and Lemma 3.3 :

"Let $b_{i}(i=1,2)$ be distinct extreme points of $B(f)$ and suppose $\left\{b_{i}\right\}$ $=F\left(D_{i}\right)$ with $D_{i} \in \mathbb{D}(i=1,2)$. Then $b_{1}$ and $b_{2}$ are adjacent in $B(f)$ if and only if the following two statements hold:

(i) There exists a common sublattice $D_{3}$ of $D_{1}$ and $D_{2}$ such that $\emptyset, E \in D_{3}$ and $\left|\Pi\left(D_{3}\right)\right|=|E|-1$.

(ii) For $T \in \Pi\left(D_{3}\right)$ with $|T|=2$ and for $S_{1}, S_{2} \in D_{3}$ with $S_{1} \subsetneq S_{2}$ and $T=S_{2}-S_{1} \quad(D, f) \cdot S_{2} / S_{1}$ is connected."

In other words, $b_{1}$ and $b_{2}$ are adjacent if and only if there exist vertices $e$ and $e^{\prime}$ in $E$ connected by an arc in both $H\left(P\left(D_{1}\right)\right)$ and $H\left(P\left(D_{2}\right)\right)$ such that the Hasse diagrams obtained by identifying $e$ with $e^{\prime}$ in $H\left(P\left(D_{1}\right)\right)$ and $H\left(P\left(D_{2}\right)\right)$, respectively, coincide with each other.

It was shown in [7] (and implicit in [5]) that any generalized polymatroid [5] is isomorphic with some base polyhedron of a submodular system which is obtained by increasing the cardinality of the ground set by one (also see [15]). Furthermore, any polymatroid polytope is a generalized polymatroid [5]. Therefore, the above characterization of adjacency for extreme points of $B(f)$ implies the result in [21]. It should also be noted that the number of adjacent extreme points of a given fixed extreme point of $B(f)$ is at most the number of edges incident with the given extreme point. Therefore, an upper bound of the number of adjacent extreme points of a given extreme point of a polymatroid polytope is given by (4.3) with $|E|$ replaced by $\left|E^{\prime}\right|+1$, where $E^{\prime}$ is the ground set of the polymatroid.

Acknowledgment: The author is grateful to one of the two referees for useful comments on the presentation of this paper.

\section{References}

[1] Bachem, A., and Grötschel, M.: New aspects of polyhedral theory. Modern Applied Mathematics - Optimization and operations Research (ed. B. Korte). North-Holland, Amsterdam, 1982, pp. $51 \sim 106$.

[2] Birkhoff, G.: Rings of sets. Duke Math. J. Vol. 3 (1937), $443 \sim 454$.

[3] Bixby, R. E., Cunningham, W. H., and Topkis, D. M.: The partial order of 
a polymatroid extreme point. Report. No. 82250-0R, Institüt fur Ökonometrie und Operations Research, Universität Bonn, December 1982.

[4] Edmonds, J.: Submodular functions, matroids, and certain polyhedra. Combinatorial Structures and Their Applications (eds. R. Guy, H. Hanani N. Sauer and J. Schönheim). Gordon and Breach, New York, 1970, pp. 69 87.

[5] Frank, A.: Generalized polymatroids. Proceedings of the Sixth Hungarian Combinatorial Colloquium, Eger, 1981 (to appear).

[6] Fujishige, S.: Principal structures of submodular systems. Discrete Applied Mathematics, Vo1. 2 (1980), $77 \sim 79$.

[7] Fujishige, S.: A note on Frank's generalized polymatroids. Discrete Applied Mathematics, Vo1. 7 (1984), $105 \sim 109$.

[8] Fujishige, S.: Submodular systems and related topics. Mathematical Programming Study (to appear).

[9] Fujishige, S., and Tomizawa, N.: A note on submodular functions on distributive lattices. Journal of the Operations Research Society of Japan, Vo1. 26 (1983), $309 \sim 318$.

[10] Giles, F. R.: Submodular functions, Graphs and Integer Polyhedra. Ph.D. Thesis, Department of Combinatorics and Optimization, University of Waterloo, Waterloo, Ontario, Canada, 1975.

[11] Iri, M.: Applications of matroid theory. Mathematical Programming - The State of the Art (eds. A. Bachem, M. Grötschel and B. Korte). Springer, Berlin, 1983, pp. $158 \sim 201$

[12] Lovász, L.: Submodular functions and convexity. Nathematical Programming - The State of the Art (eds. A. Bachem, M. Grötschel and B. Korte). Springer, Berlin, 1983, pp. $235 \sim 257$.

[13] Nakamura, M., and Iri, M.: A structural theory for submodular functions, polymatroids and polymatroid intersections. Research Memorandum RMI 8106, Department of Mathematical Engineering and Instrumentation Physics, University of Tokyo, August 1981.

[14] Rockafellar, R. T.: Convex Analysis. Princeton University Press, Princeton, N. J., 1970.

[15] Schrijver, A.: Total dual integrality from directed graphs, crossing families, and sub- and supermodular functions. Proceedings of the Silver Jubilee Conference on Combinatorics, Waterloo, 1982 (to appear).

[16] Shapley, L. S.: Cores of convex games. International Journal of Game Theory, Vo1. 1 (1971), $11 \sim 26$.

[17] Stoer, J., and Witzga11, C.: Convexity and Optimization in Finite Dimensions I. Springer, Berlin, 1970. 
[18] Tomizawa, N.: Unpublished note. July 1982 (included in [19]).

[19] Tomizawa, N.: Theory of hyperspace (XVI) - on the structures of hedrons (in Japanese). Papers of the Technical Group on Circuits and Systems, Institute of Electronics and Communication Engineers of Japan, CAS 82174, March 1983.

[20] Tomizawa, N., and Fujishige, S.: Historical survey of extensions of the concept of principal partition and their unifying generalization to hypermatroids. Systems Science Research Report No. 5, Department of Systems Science, Tokyo Institute of Technology, April 1982.

[21] Topkis, D. M.: Adjacency on polymatroids. (Unpublished manuscript.)

Satoru FUJISHIGE: Institute of Socio-Economic Planning, University of Tsukuba, Sakura, Ibaraki 305, Japan. 


\section{アブストラクト \\ 劣モジュラ・システムに関連する \\ 基多面体のすべての面の特徴づけ}

筑波大学藤 重悟

有限集合 $E$ の部分集合の族からなる分配束 $D$ とD上に定義される劣モジュラ関数 $f$ との対 ( D, $f)$ は劣モジュラ・システムと呼ばれる。

こてで, $\phi \in D, f(\phi)=0$ と仮定する。 $E \in D$ であるとき, 劣モジュラ・システム $(D, f)$ の基多面体は

$$
B(f)=\left\{x \mid x \in R^{E}, \quad \forall X \in D: x(X) \leqq f(X), x(E)=f(E)\right\}
$$

と定義される。本論文では, 基多面体 $B(f)$ の構造について考察を加え, $B(f)$ のすべての面 (faces)の特徵づけを与える。 $B(f)$ の各面は Dの固有な部分束に対応づけられ，てのような部 分束の全体 $\boldsymbol{D}$ は $B(f)$ の非空な面の全体 $\boldsymbol{F}$ に反同形である(こてで, $\boldsymbol{D}, \boldsymbol{F}$ を集合の包合関係に関 する半順序集合と考える)。Dの構造に基づいて, 面の間の接続関係, 面の次元, 面の端点执よび 端射線を与える。乙の結果は, 最近得られているとてろの(1) Bixby, Cunninghamと Topkisによ るポリマトロイド多面体の端点に関連する半順序構造と連結成分，(2)冨澤による分配束によって 定まる凸錐の端射線の特徵づけ，(3)Topkisによるポリマトロイド多面体の端点の隣接関係，の結 果を特別な場合として含む。さらに，Dの部分束D $D_{1}$ が与えられ， $f$ がD $D_{1}$ 上でモジュラであるとき，

$$
F\left(D_{1}\right)=\left\{x \mid x \in B(f), \forall X \in D_{1}: x(X)=f(X)\right\}
$$

は $B(f)$ の非空な面であり，面 $F\left(D_{1}\right)$ に対応する部分束 $D_{2} \in \boldsymbol{D}$ が一意に存在する。乙のよう な $D_{1}$ と $D_{2}$ の間の関係を特徴づける定理を示す。乙こでD $D_{2}$ は $D_{1}$ の閉包と考えることができ, この閉包は, 中村之伊理によって最近導入された最大骨格の概念と密接に関係している。以上の 各種の特徵づけについて, 算法論的な観点からも考察を加える。 